\title{
Michaelis-Menten from an In Vivo Perspective: Open Versus Closed Systems
}

\author{
Johan Gabrielsson ${ }^{1}$ and Lambertus A. Peletier ${ }^{2,3}$
}

Received 1 June 2018; accepted 16 August 2018; published online 12 September 2018

\begin{abstract}
After a century of applications of the seminal Michaelis-Menten equation since its advent it is timely to scrutinise its principal parts from an in vivo point of view. Thus, the Michaelis-Menten system was revisited in which enzymatic turnover, i.e. synthesis and elimination was incorporated. To the best of our knowledge, previous studies of the Michaelis-Menten system have been mainly based on the assumption that the total pool of enzyme, free and bound, is constant. However, in fact this may not always be the case, particularly for chronic indications. Chronic (periodic) administration of drugs is often related to induction or inhibition of enzymatic processes and even changes in the free enzymatic load per se. This may account for the fact that translation of in vitro metabolism data have shown to give systematic deviations from experimental in vivo data. Interspecies extrapolations of metabolic data are often challenged by poor predictability due to insufficient power of applied functions and methods. By incorporating enzyme turnover, a more mechanistic expression of substrate, free enzyme and substrate-enzyme complex concentrations is derived. In particular, it is shown that whereas in closed systems there is a threshold for chronic dosing beyond which the substrate concentration keeps rising, in open systems involving enzyme turnover this is no longer the case. However, in the presence of slow enzyme turnover, after an initial period of adjustment which may be quite long, the relation between substrate concentration and dose rate reduces to a linear expression. This new open framework is also applicable to transporter systems.
\end{abstract}

KEY WORDS: enzyme turnover; Michaelis-Menten kinetics; pharmacokinetics; pharmacodynamics; transporters.

\section{INTRODUCTION}

After a century since the advent of the seminal Michaelis-Menten equation, it is timely to re-examine its principal parts from an in vivo point of view (1). To the best of our knowledge, most enzyme kinetics have been studied within the context of closed systems in which the total amount of enzyme, free and bound to substrate, is effectively constant. Such systems are based on purified enzymes or on enzymes which have such a long half-life that the amount of enzyme hardly changes over the

\footnotetext{
${ }^{1}$ Department of Biomedical Sciences and Veterinary Public Health, Division of Pharmacology and Toxicology, Swedish University of Agricultural Sciences, Box 7028, SE-750 07, Uppsala, Sweden.

${ }^{2}$ Mathematical Institute, Leiden University, PB9512, 2300 RA, Leiden, The Netherlands.

${ }^{3}$ To whom correspondence should be addressed. (e-mail: peletier@math.leidenuniv.nl)
}

course of the experiment $(1-24 \mathrm{~h}) .{ }^{1}$ In light of these considerations, we associate in vitro systems with closed systems and in vivo systems with open systems.

As shown in Fig. 1, in an in vivo environment, target proteins change with half-lives ranging from less than $10 \mathrm{~h}$ to

$1 \quad$ Studying drug metabolism outside the in vivo context is most often done in vitro. If that is done with purified enzyme, no synthesis and degradation of enzyme are expected. In cell systems turnover of enzyme may still be an ongoing process. However, the time frame within which this is done is important. Even though the cell system (in vitro) may have synthesis and catabolism of enzyme, one seldom studies drug metabolism under chronic in vitro conditions. Also synthesis and catabolism are seldom measured in parallel to drug substrate metabolism. Also drug metabolism is typically studied within a time frame of a few hours or maximum a day. Enzyme half-lives are most often much longer than that so a closed system approximation may therefore be valid assuming a stable enzyme level. 


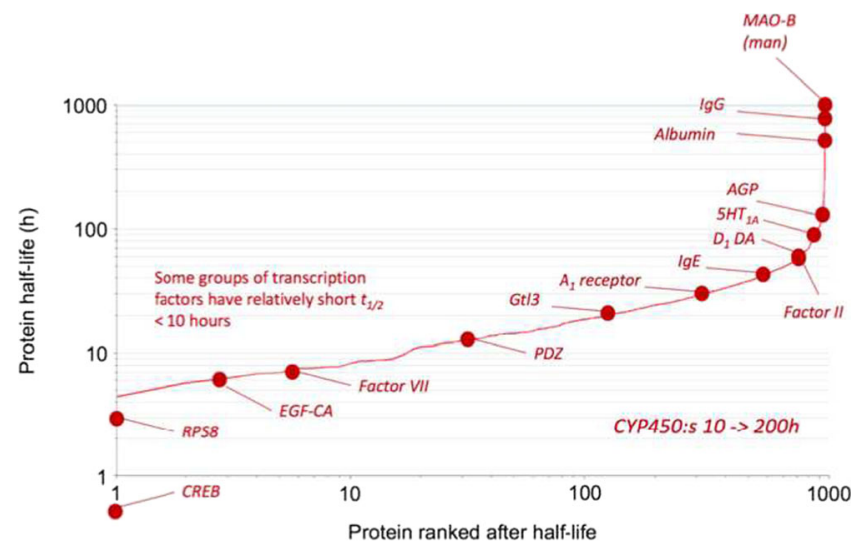

Fig. 1. Schematic presentation of half-lives of different pharmacological targets and enzymes. Some of the enzymes in the cytochrome P450 family have half-lives in the range of 10 to greater than $200 \mathrm{~h}$ (cf. $(2-7))$

greater than $1000 \mathrm{~h} \mathrm{(cf.} \mathrm{(2-6)).} \mathrm{For} \mathrm{instance,} \mathrm{the} \mathrm{important}$ cytochrome P450 family shows typically a range from about 10 to more than $200 \mathrm{~h}$ depending on isozyme (cf. von Bahr et al., (7)).

The notion of clearance employed in closed systems is commonly applied even in a physiological context for translation of in vitro metabolic data to in vivo situations in which drug-drug interactions have potential (cf RostamiHodjegan $(8,26))$. This requires a good understanding of the biological elements involved including the origin of the clearance model (open or closed). Accurate in vitro to in vivo predictions are possible when not only in vitro data are robust but also the biological structure of the clearance model is integrated.

Similar to metabolic targets, transporter proteins may also be captured by the open-system model. There is an increasing interest in the role that transporter proteins play in absorption, distribution and elimination of chemicals. Transporter proteins are significant determinants of drug disposition, drug-drug interactions and the variability seen in both absorption, disposition and pharmacological response (cf. Levy (9)). Robust information about the quantitative contribution of transporter proteins is still in its infancy, partly due to the lack of expression levels and turnover properties of the responsible protein (cf. (10-12)).

In this paper, we explore the impact of an in vivo environment on the applicability of the classical MichealisMenten model, i.e. the difference between closed and open systems as regards substrate clearance. The primary objective is to derive new expressions for the open Michaelis-Menten system and compare them to the traditional closed MichaelisMenten system. Steady state, i.e. equilibrium relationships are provided for both systems. Simulations are done with the open system showing its intrinsic behaviour. Two case studies (datasets) are then analysed by means of the open-system Michaelis-Menten model in order to show its flexibility.

Closed systems avoid the synthesis and loss of enzyme present in open systems which means that closed systems yield expressions which are less mechanistic and not optimal for translation of preclinical findings to man. We will provide insights about substrate, enzyme, substrate-enzyme complex and clearance from acute and chronic experiments.
Specifically, we show that thanks to enzyme turnover, there is no threshold to the intensity of chronic dosing and that the system eventually stabilises at a steady state which increases linearly with the input rate.

\section{THE MICHAELIS-MENTEN SYSTEM}

In Fig. 2, we show the classical system of MichaelisMenten reactions (left) as well as the 'extended' system which also incorporates enzyme turnover (right). A substrate $(S)$ binds an enzyme $(E)$ reversibly and forms a complex $E S$ which in turn generates a product $(P)$ and returns a free enzyme. The extended system includes zeroth order substrate input (Input) and enzyme turnover, as modelled in the classical indirect response model (cf. $(13,14))$ : synthesis with a zeroth order rate constant $k_{\text {syn }}$ and degradation with a first order rate constant $k_{\mathrm{deg}}$.

In mathematical terms, the open Michaelis-Menten system shown in Fig. 2 can be expressed as a system of four differential equations, one for each compound:

$$
\left\{\begin{array}{l}
\frac{d S}{d t}=k_{\mathrm{infus}}-k_{\mathrm{on}} S \cdot E+k_{\mathrm{off}} E S \\
\frac{d E}{d t}=k_{\mathrm{syn}}-k_{\mathrm{deg}} E-k_{\mathrm{on}} S \cdot E+\left(k_{\mathrm{off}}+k_{\mathrm{cat}}\right) E S \\
\frac{d E S}{d t}=k_{\mathrm{on}} S \cdot E-\left(k_{\mathrm{off}}+k_{\mathrm{cat}}\right) E S \\
\frac{d P}{d t}=k_{\mathrm{cat}} E S
\end{array}\right.
$$

where $k_{\text {infus }}=\operatorname{Input} / V_{c}$, and $k_{\text {on }} \& k_{\text {off }}$ denote the bindingand the dissociation rate of substrate-enzyme binding, $k_{\text {syn }}$ and $k_{\text {deg }}$ are the synthesis and degradation of the enzyme, $k_{\text {cat }}$ is the rate of catalysis with which the product $P$ is produced, Input is the rate at which substrate is supplied and $V$ is the distributional volume of the substrate and the enzyme. In Eq. (1), there is no loss term of product $P$ which may occur in vivo. However, the size of that clearance term requires that $\mathrm{P}$ is given as such, which is beyond the scope of this analysis.

The differential equations for the closed MichaelisMenten system are the same as those for the open system, except that the terms $k_{\mathrm{syn}}$ and $k_{\mathrm{deg}} E$ are absent.

Enzyme appears free $(E)$ and bound to the substrate $(E S)$ so that the total enzyme concentration $\left(E_{\text {tot }}\right)$ is given by

$E_{\mathrm{tot}}(t) \stackrel{\text { def }}{=} E(t)+E S(t)$

By adding the equations for free and bound enzyme from the system (1) one obtains the Enzyme conservation law:

$\frac{d}{d t} E_{\mathrm{tot}}=k_{\mathrm{syn}}-k_{\mathrm{deg}} E$

This equation shows up an important distinction between closed and open system.

- Closed system: In the closed system there is no production or loss of enzyme, i.e. $k_{\mathrm{syn}}=0$ and $k_{\mathrm{deg}}=0$, so that by Eq. (2) the total amount of enzyme, including 

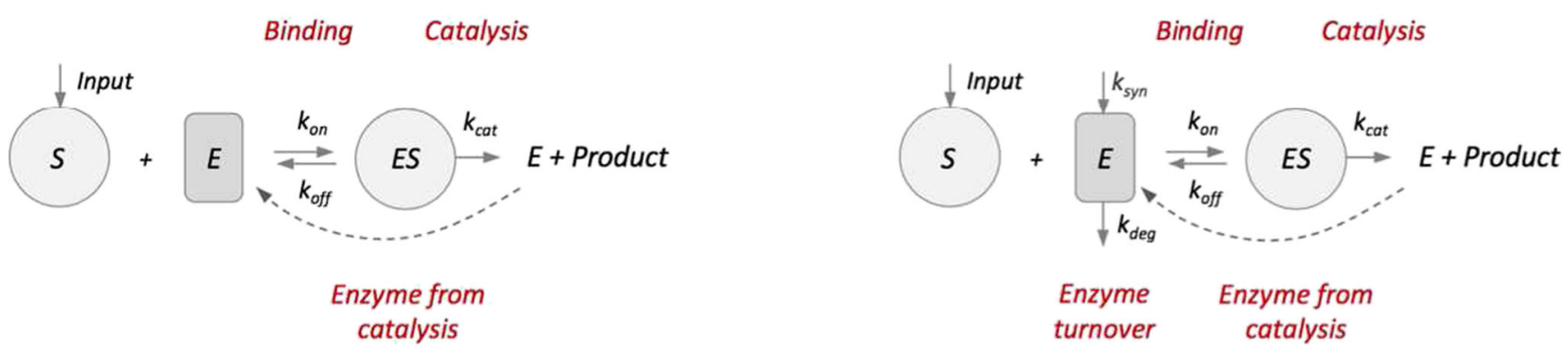

Fig. 2. Schematic description of the Michaelis-Menten model in an in vitro (closed) environment (Left) and in an in vivo (open) environment (Right), in which three processes have been added: Input of drug (Input), enzyme synthesis rate $\left(k_{\text {syn }}\right)$ and enzyme degradation (rate $\left.k_{\text {deg }}\right)(1)$

free and bound enzyme, is constant over time and stays at the initial level:

$E_{\text {tot }}(t)=E(0)+E S(0)$ for all $t \geq 0$.

Therefore, in the closed system at the steady state, the total enzyme concentration $E_{\text {tot }}$ can, in principle, take on any arbitrary value.

- Open system: Here, enzyme turnover is incorporated, i.e. $k_{\mathrm{syn}}>0$ and $k_{\mathrm{deg}}>0$. Therefore, in order for the right hand side of Eq. (2) to be zero, we must have

$E_{\mathrm{ss}}=E_{0} \stackrel{\text { def }}{=} \frac{k_{\mathrm{syn}}}{k_{\mathrm{deg}}}$

Thus, at steady state the free enzyme concentration $E_{\mathrm{ss}}$ must be equal to the baseline enzyme concentration $E_{0}$ defined in Eq. (4). Hence, here $E_{\mathrm{ss}}$ is determined by the turnover parameters only.

\section{Model Behaviour at Steady-State ${ }^{2}$}

In order to compute the steady-state concentrations, $S, E$ and ES, of the three compounds we set the time derivatives in the system (1) equal to zero. This results into three algebraic equations:

$\left\{\begin{array}{l}k_{\text {infus }}-k_{\text {on }} S \cdot E+K_{\text {off }} E S=0 \\ k_{\text {syn }}-k_{\text {deg }} E-k_{\text {on }} S \cdot E+\left(k_{\text {off }}+k_{\text {cat }}\right) E S=0 \\ k_{\text {on }} S \cdot E-\left(k_{\text {off }}+k_{\text {cat }}\right) E S=0\end{array}\right.$

We discuss the closed and the open system in succession.

- Closed systems. Subtraction of the third equation of (5) from the first one yields

In this subsection we omit the subscript 'ss'

$$
k_{\text {infus }}-k_{\text {cat }} E S=0 \Rightarrow E S=\frac{k_{\text {infus }}}{k_{\text {cat }}}=\frac{\text { Input }}{k_{\text {cat }} V}
$$

where $V$ is the distributional volume of the substrate. Hence, if the total enzyme concentration is $E_{\text {tot }}$, then

$E=E_{\text {tot }}-E S=E_{\text {tot }}-\frac{k_{\text {infus }}}{k_{\text {cat }}}$

Replacing $E$ by $E_{\text {tot }}-E S$ in the third equation of the system (5) one obtains

$S \cdot\left(E_{\mathrm{tot}}-E S\right)=K_{\mathrm{m}} \cdot E S \quad$ where $\quad K_{\mathrm{m}} \stackrel{\text { def }}{=} \frac{k_{\mathrm{off}}+k_{\mathrm{cat}}}{k_{\mathrm{on}}}$

Hence, in light of the formula for ES given by Eq. (6), the steady-state substrate concentration is given by

$$
S=K_{\mathrm{m}} \cdot \frac{k_{\text {infus }}}{k_{\mathrm{cat}} E_{\mathrm{tot}}-k_{\text {infus }}} \quad \text { if } \quad k_{\text {infus }}<k_{\text {cat }} E_{\text {tot }}
$$

Thus, for the substrate concentration to have a steady state, the infusion rate $k_{\text {infus }}$ must be bounded above by $V_{\max }$ $=k_{\text {cat }} E_{\text {tot. }}$. This upper bound is determined by the total enzyme concentration and the rate of catalysis only.

- Open systems. As we have seen in Eq. (4) and Eq. (6) which also holds for the open system, the steady-state concentrations of free and bound enzyme are given by

$E=E_{0}$ and $\quad E S=\frac{k_{\text {infus }}}{k_{\text {cat }}}$

From the third equation of the system (5) we deduce the steady-state relation 
$S \cdot E=K_{\mathrm{m}} \cdot E S, \quad K_{\mathrm{m}}=\frac{k_{\mathrm{off}}+k_{\mathrm{cat}}}{k_{\mathrm{on}}}$

As with the closed system, we substitute the expression for $E$ and $E S$ given by Eq. (10) into Eq. (11) to obtain an explicit expression for $S$ :

$S=\frac{K_{\mathrm{m}}}{E_{0}} E S=\frac{K_{\mathrm{m}}}{E_{0}} \cdot \frac{k_{\text {infus }}}{k_{\mathrm{cat}}}=\frac{\text { Input }}{\frac{k_{\mathrm{syn}}^{\prime}}{k_{\mathrm{deg}}} \cdot \frac{k_{\mathrm{cat}} \cdot k_{\mathrm{on}}}{k_{\mathrm{off}}+k_{\mathrm{cat}}}}$

where $k_{\text {syn }}^{\prime}={ }^{\text {def }} k_{\text {syn }} \cdot V$ is the synthesis rate of enzyme with units of amount per time.

Equation (12) implies that in the open system, at steady state the relation between substrate concentration $S$ and rate of Input is linear, in contrast with the relation between $S$ and Input for the closed system given by Eq. (9), which is nonlinear. This is very different from the complex steadystate relationships seen in the classical model for targetmediated drug disposition (TMDD) (cf. Gabrielsson et al. $(15,16))$. The reason is that though the two systems are similar, in the open Michaelis-Menten system at steady state $E=E_{0}$, whilst in the TMDD model the steady-state receptor concentration varies with the infusion rate (cf. (15)).

Note that when the catalytic process is much faster than the dissociation $\left(k_{\text {cat }} \gg k_{\text {off }}\right)$ Eq. (12) reduces to the simple expression

$S \approx \frac{\text { Input }}{\frac{k_{\text {syn }}^{\prime}}{k_{\mathrm{deg}}} k_{\mathrm{on}}}$

Thus, if $k_{\text {cat }} \gg k_{\text {off }}$, then the rate of catalysis no longer affects the steady-state substrate concentration.

\section{Clearance Concepts}

The advent of modern pharmacokinetics started with a seminal paper on the physiological interpretation of drug clearance by Rowland et al. (17). Their paper opened the field to a much larger and more clinically oriented audience than so far had been engaged in pharmacokinetics. Since then, numerous papers have addressed the clearance concepts from a mechanistic point of view (See Benet (18)). Below, we put the expressions derived above for the closed- and the openMichaelis-Menten system into a clearance perspective.

- Closed systems. Using the steady-state substrate concentration $S$ from (9) in the definition of clearance $C l=\operatorname{Input} / S$, we obtain here the following expression:

$C l=\frac{1}{K_{\mathrm{m}}} \cdot\left(k_{\mathrm{cat}} E_{\mathrm{tot}} V-\right.$ Input $)$

in which $E_{\text {tot }}$ is the total enzyme concentration. Using the fact that Input $=\mathrm{Cl} \cdot \mathrm{S}$ one can readily rewrite this in the more common form:
$C l=\frac{V_{\max }^{\prime}}{K_{\mathrm{m}}+S}, \quad$ where $\quad V_{\max }^{\prime}=V_{\max } \cdot V=k_{\mathrm{cat}} E_{\mathrm{tot}} \cdot V$

Interestingly, when $S \ll K_{\mathrm{m}}$ the expression for $\mathrm{Cl}$ can be well approximated by

$C l=\frac{V_{\max }^{\prime}}{K_{\mathrm{m}}}$

which traditionally denotes the intrinsic clearance based on the 'closed' system model.

Equation (15) was recently applied in a physiological context (6).

- Open systems. For open systems we deduce from Eq. (12) the following expression for the clearance $\mathrm{Cl}$ :

$C l \stackrel{\text { def Input }}{=}=\frac{k_{\text {syn }}}{k_{\mathrm{deg}}} \cdot \frac{k_{\mathrm{cat}}}{K_{\mathrm{m}}} \cdot V=\frac{k_{\mathrm{syn}}^{\prime}}{k_{\mathrm{deg}}} \cdot \frac{k_{\mathrm{cat}}}{K_{\mathrm{m}}}=\frac{V_{\mathrm{max}}^{\prime}}{K_{\mathrm{m}}}$

where $k_{\text {syn }}^{\prime}={ }^{\text {def }} k_{\text {syn }} \cdot V$ is the synthesis rate of enzyme with units of amount per time.

Thus, the traditional expression for clearance from the 'closed' system model can still be used for the 'open' system. However, in the discussion of clearance properties using the 'open' system we simply refer to 'clearance'.

The contrast between clearance in the closed system (14), which decreases with increasing infusion rate and vanishes when Input reaches $k_{\text {cat }} E_{\text {tot }} \cdot V$ and clearance in the open system (cf (18)), which is independent of the infusion rate is very obvious. This stems from the fact that whilst in the closed system the total amount of enzyme $E_{\text {tot }}$ is fixed by what it is initially and the amount of substrate-enzyme complex cannot exceed the total amount of enzyme, in the open system enzyme can be synthesised whenever the need arises.

\section{Compendium of Steady-State Expressions for S, E and ES}

For convenience, we list here the expressions for the steady-state concentrations of $S, E$ and $E S$ for the closed and the open Michaelis-Menten system (cf. Figure 2):

$$
\begin{array}{ll}
\text { Closed } & \text { Open } \\
S_{\mathrm{ss}}=K_{\mathrm{m}} \cdot \frac{k_{\text {infus }}}{V_{\text {max }}-k_{\text {infus }}}\left(k_{\text {infus }}<V_{\mathrm{max}}\right) & S_{\mathrm{ss}}=\frac{K_{\mathrm{m}}}{E_{0}} \cdot \frac{k_{\text {infus }}}{k_{\text {cat }}} \\
E_{\mathrm{ss}}=K_{\mathrm{m}} \cdot \frac{E S_{\mathrm{ss}}}{S_{\mathrm{ss}}} & E_{\mathrm{ss}}=E_{0} \\
E S_{\mathrm{ss}}=\frac{E_{0} \cdot S_{\mathrm{ss}}}{K_{\mathrm{m}}+S_{\mathrm{ss}}} & E S_{\mathrm{ss}}=\frac{k_{\text {infus }}}{k_{\mathrm{cat}}}
\end{array}
$$

where $K_{\mathrm{m}}=\left(k_{\text {off }}+k_{\text {cat }}\right) / k_{\text {on }}$ and $V_{\text {max }}=k_{\text {cat }} E_{\text {tot }}$.

\section{REAL-LIFE EXAMPLES}

In this section, we show how the open Michaelis-Menten model, given by the system (1), can be successfully fitted to 
two data sets, one for a series of iv bolus administrations and the other from a constant rate input. They have been taken from, respectively, the case studies PK38 and PK22 in Gabrielsson et al. (19).

The aim is not so much to discriminate between the 'closed' and the 'open' Michaelis-Menten system, but rather to demonstrate the flexibility and capacity of the proposed 'open' Michaelis-Menten system to handle concentration- and time-dependent kinetics. The datasets are not designed with an 'open' Michaelis-Menten system model approach, which may result in less precision of one or more parameters.

\section{Acute Experiments}

In Fig. 3, we show literature data of case study PK38 from (19) which involves elimination by two parallel Michealis-Menten systems together with an optimal fit of the open Michaelis-Menten model with enzyme turnover. This dataset shows that the proposed open system model can handle situations where otherwise empirical closed system models have been applied.

The data represents a series of acute, or single dose, experiments with increasing starting concentrations of test compound. They exhibit nonlinear behaviour which becomes more pronounced as the initial concentration increases, and the longer is the initial half-life. The final parameter estimates and their relative standard deviation $(\mathrm{CV} \%)$ are listed in Table I.

We make the following observations:

(i) The binding dissociation constant $k_{\text {off }}$ is small as compared to $k_{\text {cat }}$, and was therefore difficult to estimate precisely with the present design (CV\% greater than $4000 \%$ ).

(ii) Generally, the fit of the open Michaelis-Menten model (1) to data was good and the final parameter estimates have acceptable precision.

(iii) The model has demonstrated its flexibility to capture five concentration-time courses of substrate degradation in spite of the short observational time range and difficulties in obtaining exact and precise estimates of enzyme turnover.

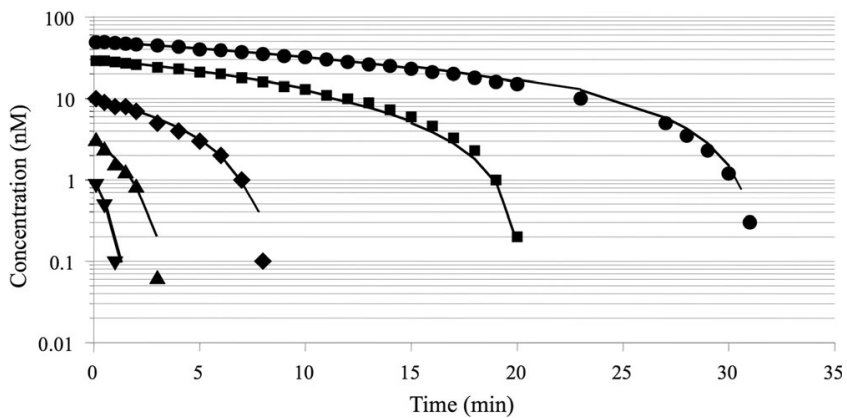

Fig. 3. Semi-logarithmic plot of observed (filled symbols) and modelpredicted (Eq. (1), solid lines) concentration-time data. The model captures accurately three orders of magnitude. Data from Gabrielsson et al. (19) case study PK38 (cf. Table I). The limit of quantification (LOQ) is about $0.1 \mathrm{nM}$. Initial concentrations are not available. It is a practical limitation of drawing samples that early
Table I. Parameter estimates for the data from PK38

\begin{tabular}{llll}
\hline Parameter & Unit & Value & CV\% \\
\hline$E_{0}$ & $\mathrm{nM}$ & 0.74 & 15.22 \\
$k_{\mathrm{deg}}$ & $1 / \mathrm{min}$ & 0.00343 & 27.95 \\
$k_{\text {on }}$ & $1 /(\mathrm{nM} \min )$ & 2.59 & 15.47 \\
$\mathrm{k}_{\text {off }}$ & $1 / \mathrm{min}$ & 0.0018 & 18,274 \\
$k_{\mathrm{cat}}$ & $1 / \mathrm{min}$ & 2.13 & 17.1 \\
$K_{\mathrm{d}}$ & $\mathrm{nM}$ & 0.00016 & - \\
$K_{\mathrm{m}}$ & $\mathrm{nM}$ & 0.69 & - \\
\hline
\end{tabular}

Remark: Since in this case study $k_{\text {cat }} \gg k_{\text {off, }}$, it follows that the expression for the clearance (10) can be simplified to become

$C l=\frac{k_{\mathrm{syn}}^{\prime}}{k_{\mathrm{deg}}} \cdot \frac{k_{\mathrm{cat}}}{K_{\mathrm{m}}} \approx \frac{k_{\mathrm{syn}}}{k_{\mathrm{deg}}} \cdot V \cdot k_{\mathrm{on}}=E_{0} \cdot V \cdot k_{\mathrm{on}}$

Equation (18) states that clearance of this test compound is directly proportional to the enzyme turnover and loss (biological properties) and the association rate constant $k_{\text {on }}$ (a physicochemical property).

\section{Repeated Intravenous Infusions}

The open Michaelis-Menten model shown in Fig. 2 was also fitted to literature data (case study PK22, Gabrielsson et al. (19)) in order to challenge the open system model following repeated iv infusions over an observational period of almost $100 \mathrm{~h}$ (cf. Fig. 4).

This is a dataset that previously was analysed with a closed system auto-induction model (cf Levy (20,21) and Abramson (24,25)). The final parameter estimates are given in Table II.

We make the following observations

(i). The open Michaelis-Menten model shown in Fig. 2 and (1) has demonstrated its ability to capture a

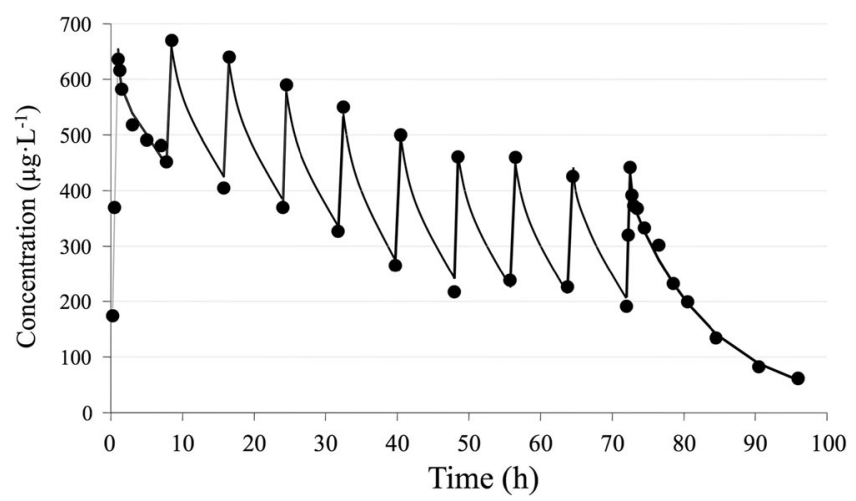

Fig. 4. Observed (filled symbols) and model-predicted (Eq. (1), solid lines) concentration-time data after repeated dosing of a test compound. The model captures accurately the complete concentration-time course. Data obtained from Gabrielsson et al. (19), case study PK22. Limit of quantification LOQ is $10 \mu \mathrm{g} / \mathrm{L}$. The first iv infusion dose was $120 \mathrm{mg}$ and given for 1 hour. The following nine doses were $40 \mathrm{mg}$ each and given as $30 \mathrm{~min}$ constant iv infusions at $8,16,24,32,40,48,56,64$ and $72 \mathrm{~h}$ 
Table II. Parameter estimates for the data from PK22

\begin{tabular}{llcl}
\hline Parameter & Unit & Value & CV\% \\
\hline$E_{0}$ & $\mu \mathrm{g} / \mathrm{L}$ & 20.6 & 36 \\
$k_{\mathrm{deg}}$ & $1 / \mathrm{h}$ & 1.30 & 47 \\
$k_{\text {on }}$ & $1 /(\mu \mathrm{g} / \mathrm{L} \cdot \mathrm{h})$ & 0.011 & 26 \\
$k_{\text {off }}$ & $1 / \mathrm{h}$ & 0.12 & 47 \\
$k_{\text {cat }}$ & $1 / \mathrm{h}$ & 0.13 & 29 \\
$K_{\mathrm{d}}$ & $\mu \mathrm{g} / \mathrm{L}$ & 11 & - \\
$K_{\mathrm{m}}$ & $\mu \mathrm{g} / \mathrm{L}$ & 23 & - \\
\hline
\end{tabular}

concentration-time course of substrate after repeated dose administration without incorporating timedependent decrease in $k_{\text {deg }}$ (or increase in $k_{\text {syn }}$ ) to mimic auto-induction.

(ii). Some of the parameters were estimated with poor precision, including the binding dissociation constant $k_{\text {off }}$, but the experiment was designed with a closed system-induction model in mind.

(iii). Steady state seems to have been reached at about $50 \mathrm{~h}$. The values of $K_{\mathrm{d}}, K_{\mathrm{m}}$ and $V_{\text {max }}=k_{\text {cat }} E_{0} V$ are estimated at $11 \mu \mathrm{g} / \mathrm{L}$ and $23 \mu \mathrm{g} / \mathrm{L}$, respectively, by means of the open system model.

Theoretically, the ratio of $V_{\max } / K_{\mathrm{m}}$ is viewed as intrinsic clearance of a compound. In this particular example, the analysis relies on peripheral venous plasma and not drug concentration at the enzyme site, which means that $V_{\max } / K_{\mathrm{m}}$ will correspond to plasma clearance.

\section{DYNAMICAL SYSTEMS BEHAVIOUR}

We discuss the dynamics of the open substrate-enzyme system as shown in Fig. 2 and mathematically in the system (1). We do this for two types of dosing: (i) Acute time courses and (ii) Constant-rate input.

\section{Acute Dosing}

The classical Michaelis-Menten equation of clearance of substrate $(S)$ and the rate of metabolism (Rate),

$$
C l=\frac{V_{\max }}{K_{\mathrm{m}}+S} \quad \text { and } \quad \text { Rate }=C l \times S=\frac{V_{\max }}{K_{\mathrm{m}}+S} \times S,
$$

are typically used to capture the dynamic behaviour of the substrate concentration $(S)$ over a range of different substrate concentrations. As the dose of the substrate increases, the clearance decreases resulting in a nonlinear graph of the substrate-time course. In particular, the half-life $\left(t_{1 / 2}\right)$ is seen to increase accordingly. However, the equations for clearance and rate shown in (19) were derived for a closed substrateenzyme system.

In this section, we show that the same substrate-enzyme binding, but then placed in an open system (cf. Fig. 2 and the system (1)) exhibits comparable temporal nonlinearities such as shown in Fig. 3, but with critical differences.

In order to uncover the characteristic properties of the dynamics of the open system, we show in Fig. 5 a series of simulations of the temporal behaviour of substrate $S(t)$, enzyme $E(t)$ and complex $E S(t)$ and the latter two combined $E_{\mathrm{tot}}(\mathrm{t})=E(t)+E S(t)$ after a series of bolus dose administrations of substrate, where the doses have been chosen so that $S_{0}=0.1,0.3,1.0,3.0,10.0,30.0,50.0$. It is assumed that initially the system is free of substrate, i.e. $E S=0$ and that the enzyme concentration is at its baseline value $E_{0}=k_{\mathrm{syn}} / k_{\mathrm{deg}}$.

We make the following observations:

- Initially, substrate-enzyme binding occurs rapidly due to the second-order process: the concentration of free enzyme drops steeply, of enzyme-substrate complex drops fast and of substrate drops accordingly.

- The substrate concentration-time courses exhibit the typical nonlinear behaviour, especially for higher doses in which clearance increases as the substrate concentration decreases.

- The free-enzyme concentration displays prolonged suppression over the period of largesubstrate concentration, which lasts longer as the substrate dose increases. Subsequently, it rebounds and displays an overshoot before it converges towards the steady state value $E_{0}$.

- The time-courses substrate complex reveal interesting patterns particularly for the higher substrate doses. An initial rapid jump up to about $E_{0}=1$ for the higher doses is followed by a gradual monotone build-up of substrate-enzyme complex which suddenly drops off when substrate concentrations drops to $O\left(K_{\mathrm{m}}\right)$.

- The total enzyme concentration is seen to increase linearly up to the time that $S$ has dropped to values of $O\left(K_{\mathrm{m}}\right)$ and enzyme is released and rapidly increases and enzyme-substrate complex drops off, i.e. there is a rapid dissociation of enzyme and substrate. When $S_{0}=50$ then at its maximum the total enzyme concentration is seen to have grown to about $140 \%$ of its initial value.

In Fig. 6, we show comparable concentration graphs of $S$, $E$ and $E S$ for the closed system, i.e. when $k_{\mathrm{syn}}=0$ and $k_{\mathrm{deg}}=0$, on a linear concentration scale. The different behaviour of the free-enzyme concentration is evident. But the reduced substrate clearance shows up in the substrate graphs where the times when $S$ has dropped down to values of $O\left(K_{\mathrm{m}}\right)$ are noticeably larger than in the open system shown in Fig. 5.

\section{Constant Rate Input}

As shown in the section "THE MICHAELIS-MENTEN SYSTEM", when drug is supplied through a constant rate infusion $k_{\text {infus }}$, the steady-state concentrations of the three compounds are given by 

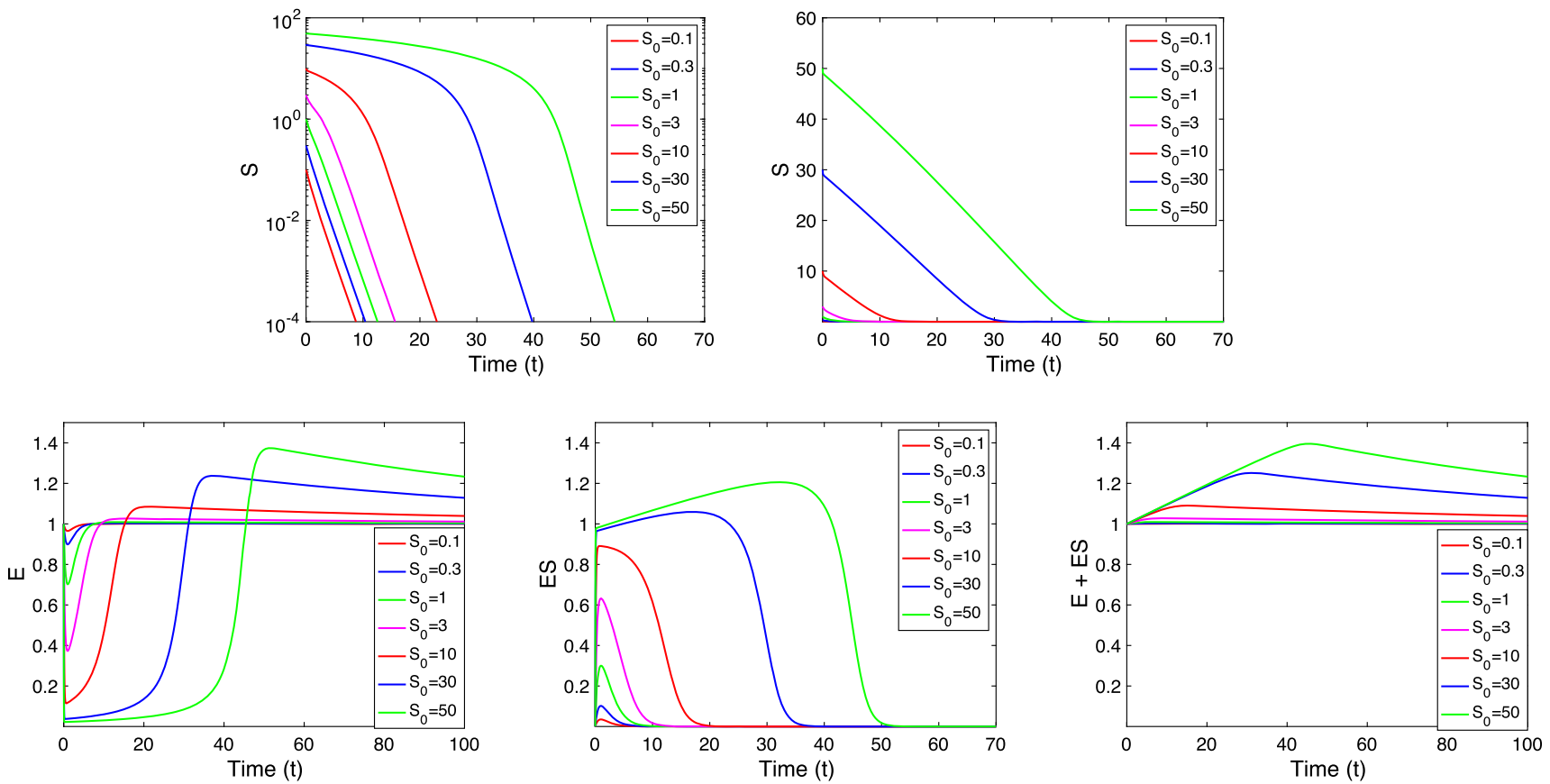

Fig. 5. Acute doses in the open system: Log-linear and linear graphs of $S(\mathrm{t})$ (top left and top right) and linear graphs of $E(t)$ (bottom left) and ES $(t)$ (bottom middle); total enzyme concentration $E_{\text {tot }}(t)$ (bottom right) for seven initial substrate concentrations $S(0)=0.1,0.3,1.0,3.0$, $10.0,30.0$ and 50.0, for $E(0)=1$ and for $E S(0)=0$. The parameters are taken from Table III

$S_{\mathrm{ss}}=\frac{1}{E_{0}} \cdot \frac{K_{\mathrm{m}}}{k_{\mathrm{cat}}} \cdot k_{\text {infus },} \quad E_{\mathrm{ss}}=E_{0}, \quad E S_{\mathrm{ss}}=\frac{k_{\text {infus }}}{k_{\text {cat }}}$.

Thus, at steady state $S$ and $E S$ increase linearly with the infusion rate, whilst $E$ is independent of $k_{\text {infus }}$.

In Fig. 7, we vary the infusion rate choosing $k_{\text {infus }}=0.01$, $0.03,0.1,0.3,1.0,3.0$, and keep the initial values constant: $(S$, $E, E S)(0)=\left(0, E_{0}, 0\right)$. The graphs show that both substrate and enzyme concentrations exhibit overshoot: Substrate immediately and enzyme after a delay that increases as the dose increases

The graphs are similar to those shown for the single-dose simulations shown in Fig. 5: at the higher infusion rates, washout is nonlinear, enzyme suppression is pronounced for high-infusion rates, displays overshoot and returns to baseline $E_{0}$. However, there are a few distinct new features:

- At the higher infusion rates, enzyme suppression extends well beyond the infusion period.
- The substrate-enzyme complex gradually reaches steady state at the lower drug input rates. At the higher rates, the substrate-enzyme complex quickly increases towards the baseline value $E_{0}$ and then continues to increase in a more or less linear fashion well beyond the time the drug input is stopped.

\section{Mathematical Analysis}

We discuss the dynamics of the open substrate-enzyme system following two types of dosing: (i) Acute dosing and (ii) dosing through a constant-rate infusion. We shall give a qualitative and quantitative analysis of the impact of enzyme turnover for these two kinds of dosing. In the analysis of acute dosing, we make the following assumptions:

(1) The total enzyme concentration is assumed to be small compared to the substrate concentration, i.e. $E_{0} \ll S_{0}$.
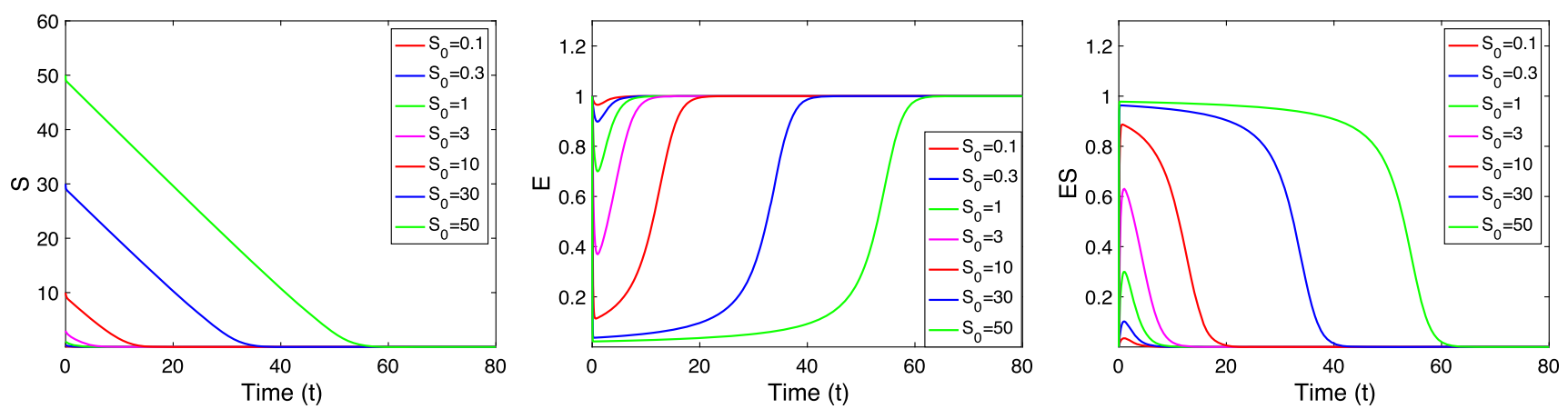

Fig. 6. Acute doses in the closed system: Linear graphs of $S(t), E(t)$ and $E S(t)$ for initial data as in Fig. 5. The parameters are taken from Table III 

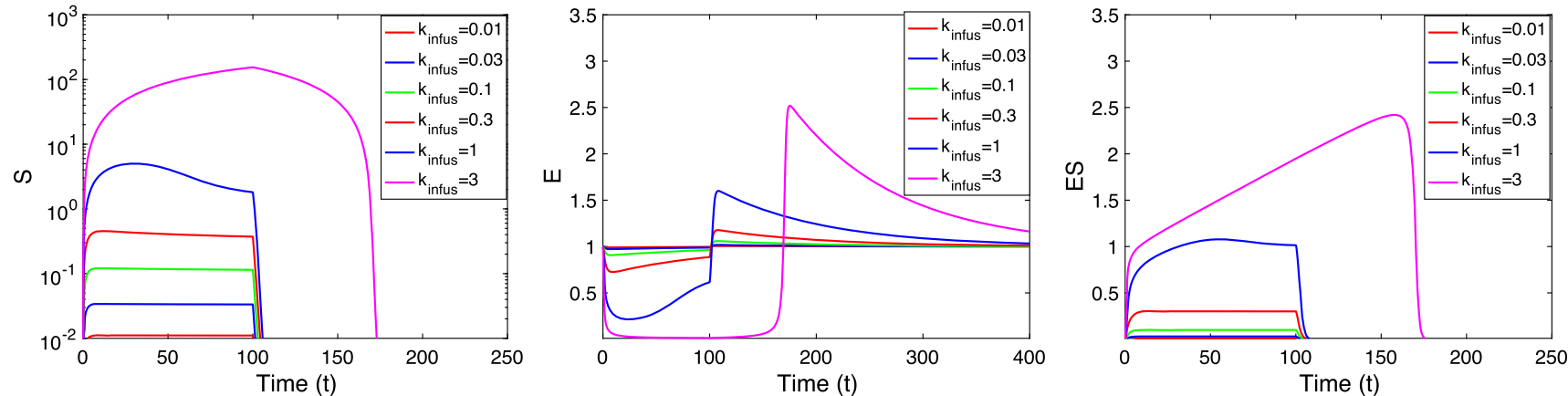

Fig. 7. Constant rate input over 100 min: Log-linear graphs of $S$ (t) (left), linear graphs of $E$ (t) (middle) and ES (t) (right) for six constant rate substrate-infusions rates: $k_{\text {infus }}=0.01,0.03,0.1,0.3,1.0,3.0$; with initial values $S(0)=0, E(0)=1$ and $\operatorname{ES}(0)=0$. The parameters are taken from Table III

(2) Enzyme turnover is assumed to be slow compared to the binding dynamics, specifically $k_{\mathrm{deg}} \ll k_{\mathrm{on}} S_{0}$.

\section{Acute Dosing}

The simulations of the dynamics after the iv bolus administration, in Fig. 5, suggest that for higher initial substrate concentrations and rapid binding, as in TMDD (22), the dynamics proceeds in distinct temporal phases: (i) a brief initial phase in which substrate binds the enzyme (phase A); then (ii) a longer phase in which $S \gg K_{\mathrm{m}}$ and so the enzyme is more or less saturated and the free-enzyme concentration is small (phase B); then (iii) a transitional phase in which $S=O\left(K_{\mathrm{m}}\right)$ (phase C) and finally (iv) a terminal phase in which $S \rightarrow 0$ (phase D). In Fig. 8, we give a schematic overview.

Below we briefly describe the four phases:

Phase A: Immediately after the iv bolus dose, substrate binds quickly to the enzyme over a period of time that is short compared to that of enzyme turnover. Specifically, the half-life of that binding process amounts to about $t_{1 / 2}=\ln (2) /$ $\left(k_{\mathrm{on}} S_{0}\right) \min$ (cf. Appendix A for the derivation of this estimate.). For the parameter values of Table III, this amounts to $t_{1 / 2}=$ $0.07 \mathrm{~min}$. Since this phase is so short, the substrate concentration and the total enzyme concentration hardly change so that at the end of this phase, substrate, enzyme and complex are in quasiequilibrium. Therefore, at the end of phase A the concentrations are well approximated by

$S=S_{0}, \quad E=E_{0} \cdot \frac{K_{\mathrm{m}}}{K_{\mathrm{m}}+S_{0}} \quad$ and $\quad E S=E_{0} \cdot \frac{S_{0}}{K_{\mathrm{m}}+S_{0}}$

If $S_{0} \gg K_{\mathrm{m}}$, then these approximate initial conditions for phase $\mathrm{B}$ reduce to

$S=S_{0}, \quad E=0 \quad$ and $\quad E S=E_{0}$

In understanding the dynamics in the remaining phases, the conservation laws for the substrate and enzyme will be useful. For convenience we recall them here:

$$
\begin{aligned}
& \frac{d}{d t}(S+E S)=-k_{\mathrm{cat}} E S \quad \text { Substrate } \\
& \frac{d}{d t}(E+E S)=k_{\mathrm{syn}}-k_{\mathrm{deg}} E \quad \text { Enzyme }
\end{aligned}
$$

Phase B: $\left(S \gg K_{\mathrm{m}}\right)$ in this phase the enzyme is more or less saturated and hence $E \approx 0$. Therefore, the enzyme conservation law can be approximated by

$\frac{d E S}{d t}=k_{\mathrm{syn}}$

At the transition from phase A to phase B, we have $E S=$ $E_{0}$ by (21). Since phase A is very short, we may put $E S(0)=$ $E_{0}$ so that together with Eq. (24) we obtain

$E S(t)=E_{0}+k_{\mathrm{syn}} \cdot t$

By using Eq. (24), together with the expression for ES from (25), in the substrate conservation law (23) we obtain

$\frac{d S}{d t}=-k_{\mathrm{syn}}-k_{\mathrm{cat}}\left(E_{0}+k_{\mathrm{syn}} \cdot t\right)$

or when we separate the terms due to enzyme turnover,

$\frac{d S}{d t}=-k_{\mathrm{cat}} E_{0}-k_{\mathrm{syn}}\left(1+k_{\mathrm{cat}} \cdot t\right) \quad$ if $\quad S \gg K_{\mathrm{m}}$

Therefore, the temporal behaviour of the substrate concentration, when it is large enough, is given by

$S(t)=S_{0}-\left(k_{\mathrm{cat}} E_{0}+k_{\mathrm{syn}}\right) \cdot t-\frac{1}{2} k_{\mathrm{cat}} k_{\mathrm{syn}} \cdot t^{2}$

The terms involving $k_{\text {syn }}$ owe their presence to the enzyme turnover. Thus, as a result of enzyme turnover, clearance is larger than in the closed system. 


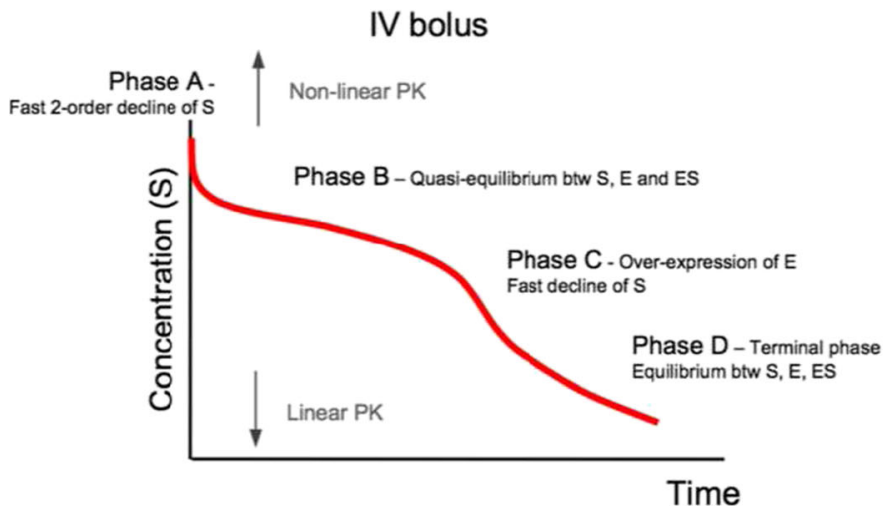

\section{Constant IV input}

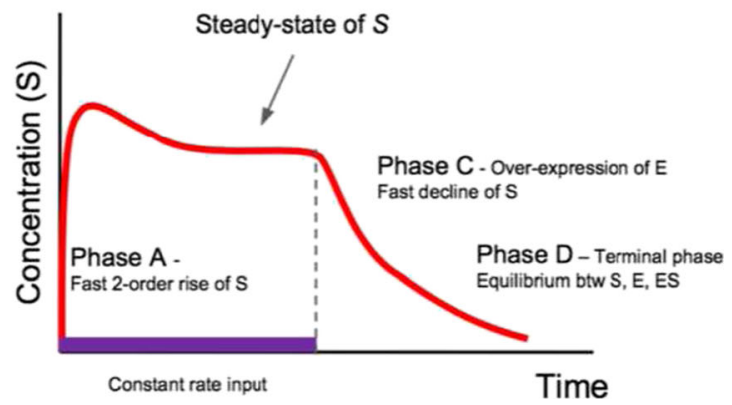

Fig. 8. Schematic presentation of the phases A to D seen in the disposition of substrate $S$ after an intravenous bolus dose (left) and a constantrate infusion (blue bar) (right). The left hand pattern is similar to the time course of drug that obeys traditional target-mediated drug disposition (22)

Clearly, according to Expression (28) for $S(t)$, it follows that as $t$ becomes large then $S(t)$ becomes small and hence we leave phase $\mathrm{B}$ to enter phase $\mathrm{C}$.

Phase C: $\left(S=O\left(K_{\mathrm{m}}\right)\right)$. As seen in Fig. 5, upon entering phase $\mathrm{C}$, the substrate-enzyme complex dissociates and the free enzyme becomes available. Since during phase B enzyme has been synthesised at the rate of $k_{\text {syn }}$ and very little has been degraded, it follows that $E$ overshoots the concentration $E_{0}$ which it had at the start of dosing. Clearly, the amount of overshoot increases as the length of phase B increases.

Phase D: Terminal phase. As $S(t) \rightarrow 0$ we enter the terminal phase. The nonlinear system (1) can now be linearised and the half-life of the three compounds can be computed explicitly. For the data of Table III, one finds $t_{1 / 2}=$ 70 min, which agrees with the findings of Fig. 7. For details of this derivation, we refer to Appendix B.

\section{Constant-Rate Input}

As it is well known about the closed system, for smaller infusion rates: $k_{\text {infus }}<V_{\max }=k_{\text {cat }} E_{0}$, the substrate concentration $S$ converges to a steady state $S_{\mathrm{ss}}$ (cf. Eq. (9)), but for rates in excess of $V_{\max }$ no steady state exists and $S$ grows indefinitely.

In the open system this is no longer so and, as shown in the section THE MICHAELIS-MENTEN SYSTEM, for every $k_{\text {infus }}>0$ there exist a steady-state concentration. In Fig. 9, we see how the three compounds all converge to a steady state as $t \rightarrow \infty$. For the values of Table III, they are given by

$S_{s s}=1.1 \times k_{\text {infus },} \quad E_{\mathrm{ss}}=1, \quad E S_{\mathrm{ss}}=k_{\text {infus }}$,

which is confirmed by the simulations shown in Fig. 9.

When $k_{\text {infus }} \geq V_{\text {max }}$ in the open system, additional enzyme can be produced. However, because of the slowenzyme turnover this takes time. For $k_{\text {infus }}$, large enough, $S$ reaches values $S \gg K_{\mathrm{m}}$, i.e. values which hold in phase B. Following the analysis done for this phase, we conclude that $S(t)$ can be approximated by
$S(t)=\left\{k_{\text {infus }}-\left(k_{\text {cat }} E_{0}+k_{\text {syn }}\right)\right\} \cdot t-\frac{1}{2} k_{\mathrm{cat}} k_{\mathrm{syn}} \cdot t^{2}$

We see this confirmed by the simulations in Fig. 9 for six infusion rates over a period that is ten times as long as in Fig. 7. In the top left graph of Fig. 9, the parabolic shape of $S$ $(t)$ is clearly apparent for the highest dose: $k_{\text {infus }}=3$.

According to Eq. (29), the positive zero $t=t_{0}$ of the graph of $S(t)$ is given by

$t_{0}=2 \times \frac{k_{\text {infus }}-\left(k_{\text {cat }} E_{0}+k_{\text {syn }}\right)}{k_{\text {cat }} k_{\text {syn }}}=398$ for $\quad k_{\text {infus }}=3$.

Once $S$ is small $\left(S \ll K_{\mathrm{m}}\right)$, as in phase C, the substrateenzyme complex dissociates resulting in a rebound of the free-enzyme concentration. After the rebound the system enters phase $\mathrm{D}$ in which the three concentrations are seen to slowly converge to their respective steady states with a halflife determined by the terminal slope $\lambda_{\mathrm{z}}=k_{\mathrm{deg}}$, which here amounts to $69 \mathrm{~h}$, as shown in Appendix B.

\section{TRANSPORTERS}

There is an increasing interest in the role that transporter proteins play in absorption, distribution and elimination of chemicals (See Pang et al. (10) for an overview). Transporter proteins are also significant determinants of drug disposition, drug-drug interactions and the variability seen in both absorption, disposition and pharmacological response (Levy (9)). Levy noted that the potency $E S_{50}$ will increase or decrease depending on inter-individual differences in maximum transport capacity $V_{\max }$, the transporter MichaelisMenten constant $K_{\mathrm{m}}$ or both. Robust information about the quantitative contribution of transporter proteins is still in its

Table III. Parameter values used in Figs. 5, 6, 7, 9 and 11

\begin{tabular}{lllllll}
\hline Parameter & $k_{\text {on }}$ & $k_{\text {off }}$ & $k_{\text {cat }}$ & $k_{\text {deg }}$ & $K_{\mathrm{d}}$ & $K_{\mathrm{m}}$ \\
\hline Value & 1 & 0.1 & 1 & 0.01 & 0.1 & 1.1 \\
\hline
\end{tabular}



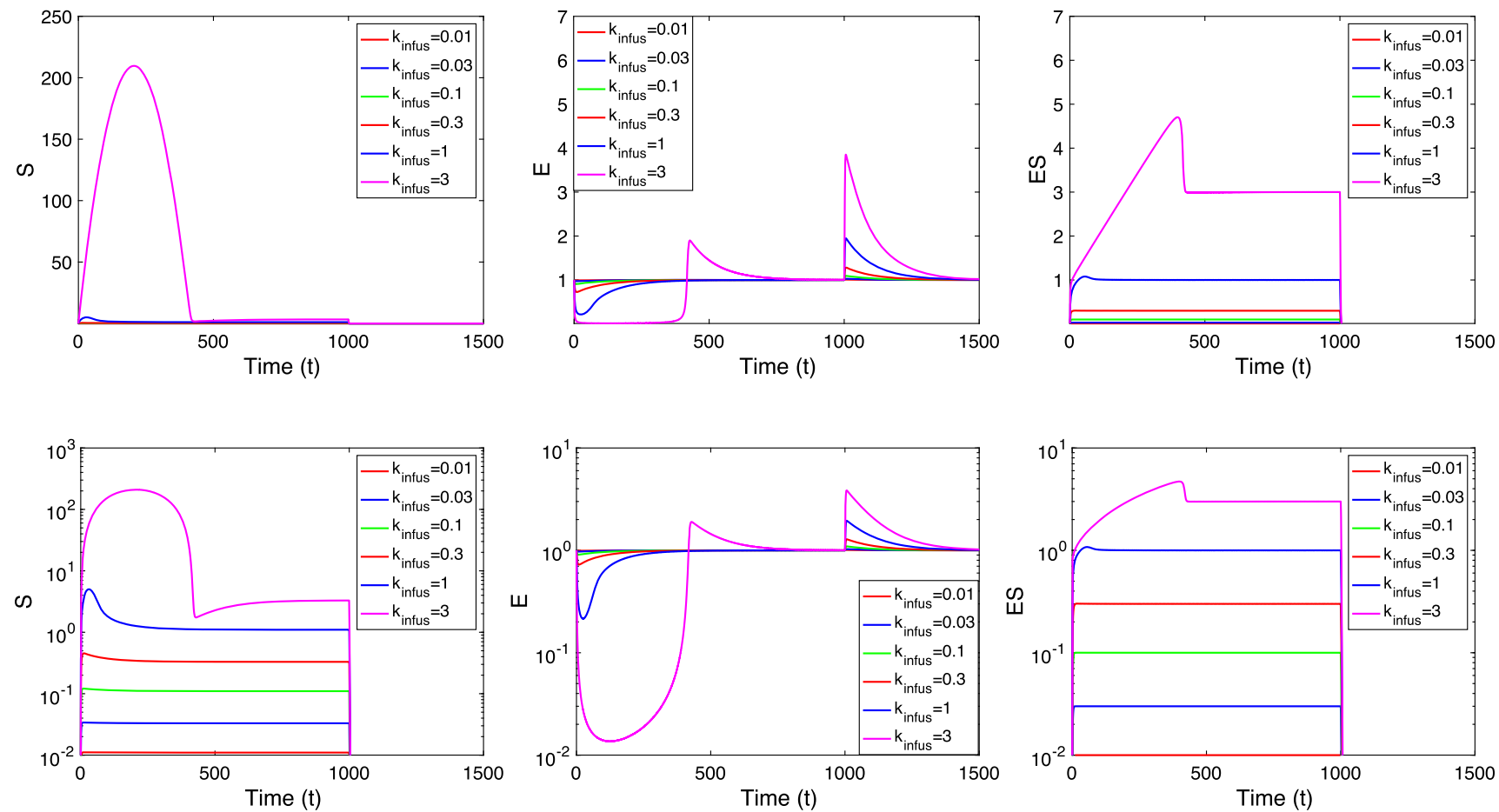

Fig. 9. Constant-rate infusion over $1000 \mathrm{~min}$ into the open system. At the top: linear graphs of $S(t)$ (left), $E(t)$ (middle) and $E S(t)$ (right) for six constant-rate substrate infusion rates: $k_{\text {infus }}=0.01,0.03,0.1,0.3,1.0,3.0$; with initial values $S(0)=0, E(0)=1$ and $E S(0)=0$. At the bottom: the same graphs but concentrations are measured along a logarithmic scale. The parameters are taken from Table III

infancy, partly due to the lack of expression levels and turnover properties of the responsible protein. The goal of this section is to highlight some potential properties and similarities of an open system-transporter model shown in Fig. 10.

The rate equations describing the transporter system shown in Fig. 10 are

$$
\left\{\begin{array}{l}
\frac{d S}{d t}=k_{\text {infus }}-k_{\mathrm{on}} S \cdot T+k_{\mathrm{off}} T S \\
\frac{d T}{d t}=k_{\mathrm{syn}}-k_{\mathrm{deg}} T-k_{\mathrm{on}} S \cdot T+\left(k_{\mathrm{off}}+k_{\mathrm{trans}}\right) T S \\
\frac{d T S}{d t}=k_{\mathrm{on}} S \cdot T-\left(k_{\mathrm{off}}+k_{\text {trans }}\right) T S \\
\frac{d P}{d t}=k_{\text {trans }} T S
\end{array}\right.
$$

where $k_{\text {infus }}=$ Input $/ V$ and $k_{\text {trans }}$ is the first-order transporter rate constant.

Despite considerable evidence about the importance of transporters in absorption and disposition of drugs, knowledge of the basic turnover properties of transporter proteins is still rudimentary. It is therefore suggested that in light of the important findings about the open Michaelis-Menten system, the in silico-transporter models ought to be built as open systems. The proposed transporter model (Fig. 10) has potential to mimic iv bolus plasma data of for example oligonucleotide and antibody kinetics (Geary et al. (23)). The extreme plasma concentration-time profile of oligonucleotides, with a dramatic initial drop with respect to rate and extent, followed by a much slower terminal decline, may be captured by a high-capacity pool of transporter protein exceeding the oligonucleotide dose. This has been demonstrated for some target-mediated drug disposition systems (cf. $(15,22))$.

\section{OVERALL CONCLUSIONS}

\section{Open vs. Closed Systems}

An important advantage of using the open system approach is that one does not have to mimic an apparent

\section{Open transporter system (in vivo)}

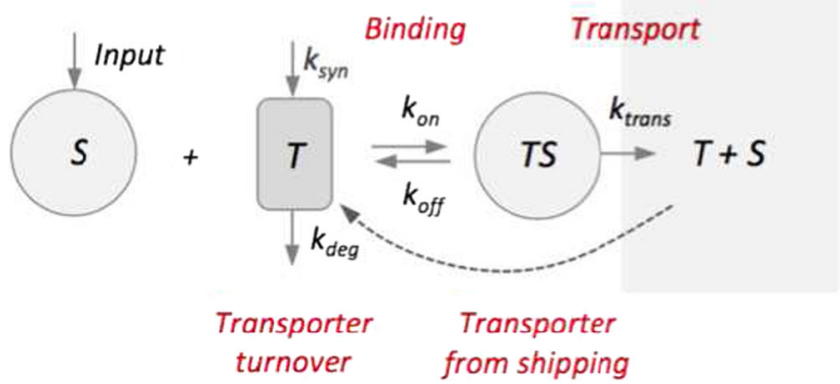

Fig. 10. Schematic diagram of an in vivo (open) transporter system where free substrate $S$ is transported from, e.g. blood/plasma into the cell (shaded area). Here In, $S, T$ and $T S$ denote substrate input rate, substrate, transporter and substrate-transporter complex, respectively. The turnover parameters of the transporter protein $k_{\text {syn }}$ and $k_{\mathrm{deg}}$, and the binding parameters $k_{\text {on }}$ and $k_{\text {off }}$. The parameter $k_{\text {trans }}$ denotes the first-order transport rate across the cell wall 
temporal nonlinearity, for instance in the clearance, by means of a saturable expression such as shown in Eq. (19).

In open systems, the nonlinearity observed for iv bolus dosing, in which for a period of time clearance decreases and half-life increases, is an intrinsic property of the system which results from the dynamic relation between substrate $S$, free enzyme E and substrate-enzyme complex ES. Specifically, at steady-state clearance is given by the expression (cf. Eq. (9))

$C l=\frac{k_{\text {syn }}^{\prime}}{k_{\text {deg }}} \times \frac{k_{\text {cat }} \cdot k_{\text {on }}}{k_{\text {off }}+k_{\text {cat }}}$

Therefore, in open systems clearance at steady state is independent of the substrate concentration and hence of the infusion rate.

This contrasts with clearance in closed systems which does depend in the infusion rate, as shown in (14):

$C l=\frac{1}{K_{\mathrm{m}}} \cdot\left(k_{\mathrm{cat}} \frac{k_{\mathrm{syn}}^{\prime}}{k_{\mathrm{deg}}}-\right.$ Input $)$

When we use the relation Input $=C l \cdot S$ to replace Input by $S$ in Eq. (33), we obtain the following formula for the clearance:

$C l=\frac{V_{\max }^{\prime}}{K_{\mathrm{m}}+S}$

From a practical point of view, the Expression (32) is useful since it reveals the intrinsic properties of clearance in an open system; both the biological properties $k_{\text {syn }}$ and $k_{\text {deg }}$, and the physico-chemical properties $k_{\text {off }}, k_{\text {on }}$ and $k_{\text {cat }}$. Since $S$, $E$ and $E S$ return to their steady-state values for large times, the expressions for these steady state values show that $E$ returns to baseline $E_{0}$, substrate-enzyme complex to $k_{\text {infus }} / k_{\text {cat }}$ and substrate to $\left(K_{\mathrm{m}} / V_{\max }\right) \times k_{\text {infus. }}$.

\section{Irreversible Systems}

When the system is essentially irreversible, i.e. when $k_{\text {cat }} \gg k_{\text {off }}$ then at steady state the clearance formula simplifies considerably. Recall from (10) that the full expression of clearance is

$C l=\frac{k_{\mathrm{syn}}^{\prime}}{k_{\mathrm{deg}}} \times \frac{k_{\mathrm{cat}} \cdot k_{\mathrm{on}}}{k_{\mathrm{off}}+k_{\mathrm{cat}}}$

Thus, if $k_{\text {cat }} \gg k_{\text {off, }}$, the expression for the clearance simplifies to

$C l=\frac{k_{\mathrm{syn}}^{\prime}}{k_{\mathrm{deg}}} \cdot k_{\mathrm{on}}$,

i.e. the clearance can be expressed as the product of the equilibrium amount of enzyme times the substrate-enzyme binding rate.

\section{Final Remarks}

The analysis in this paper has demonstrated the flexibility and capacity of the proposed 'open' Michaelis-Menten system to handle concentration- and time-dependent kinetics without the obvious limitations of the traditional closed system Michaelis-Menten model.

The duality of the acute versus equilibrium states of the open system model is also discussed, in that the free enzyme dynamics continuously governs the clearance of a drug until equilibrium is reached. The free-enzyme concentration is governed by enzyme synthesis and degradation, catabolism and regeneration of free enzyme from the substrate-enzyme complex as product $P$ is formed.

Substrate $S$ and product $P$ in Fig. 2 typically have clearances, $C l_{\mathrm{S}}$ and $C l_{\mathrm{P}}$, respectively as well. These additional clearing processes can easily be included in the model and put into the system (1). This yields expressions for the steady states of $S, E$ and $E S$ and offers a way to give a quantitative estimate of the impact of these clearances on the dynamics. Here, we have refrained from doing so in order to more clearly demonstrate the differences between the original closed system and the open system without making the comparisons too complicated.

An interesting observation made in this analysis of the open system is that whilst the system of equations governing the dynamics of the open system are clearly nonlinear, the steady-state concentration of $S$ and $E S$ depend linearly on the infusion rate. This observation has changed our view of how to assess and model nonlinear pharmacokinetics regardless of the source (time or capacity).

\section{APPENDIX A SHORT-TIME BEHAVIOUR AFTER ACUTE DOSING}

In order to estimate the different time scales in the dynamics of the 'open' Michaelis-Menten system (1) we introduce dimensionless variables in which we scale the variables $S, E$ and $E S$ by characteristic values. In this case, natural choices for these values are the initial values of $S$ and $E$, which we put $S(0)=S_{0}, E(0)=E_{0}=k_{\mathrm{syn}} / k_{\mathrm{deg}}$ and $E S(0)=0$. As it turns out, a natural choice for a characteristic time will be $1 /\left(k_{\mathrm{on}} E_{0}\right)$. Thus, we define the dimensionless concentrations

$x(\tau)=\frac{S(t)}{S_{0}}, \quad y(\tau)=\frac{E(t)}{E_{0}} \quad z(\tau)=\frac{E S(t)}{E_{0}}$,

and the dimensionless time $\tau=k_{\mathrm{on}} E_{0} \cdot t$. Then,

$x(0)=1, \quad y(0)=1, \quad z(0)=0$

Introducing these variables into the first three equations of (1) we obtain

$\left\{\begin{array}{l}\frac{d x}{d \tau}=\rho-x \cdot y+v \cdot z \\ \varepsilon \frac{d y}{d \tau}=\sigma(1-y)-x \cdot y+k \cdot z \\ \varepsilon \frac{d z}{d \tau}=x \cdot y-k \cdot z\end{array}\right.$ 
where the dimensionless parameters $\varepsilon, \kappa, v$ are defined by

$\varepsilon=\frac{E_{0}}{S_{0}}, \quad k=\frac{K_{\mathrm{m}}}{S_{0}}, \quad v=\frac{K_{\mathrm{d}}}{S_{0}}$

and the dimensionless flow rates $\sigma$ and $\rho$ by:

$\rho=\frac{k_{\text {infus }}}{k_{\text {on }} S_{0} \cdot E_{0}}$ and $\sigma=\frac{k_{\text {deg }}}{k_{\text {on }} S_{0}}$

\section{Assumptions}

(i) As is common in the classical Michaelis-Menten theory, we focus on the situation when the enzyme concentration is small compared to the substrate concentration, i.e. $E_{0} \ll S_{0}$. In terms of the dimensionless parameters, we thus focus on the case when $\varepsilon \ll 1$.

(ii) We assume that enzyme turnover is slow compared with the half-life of the initial phase, i.e. we assume that $\sigma \ll 1$.

In order to concentrate on the initial behaviour, we blow up time and write $\tau_{1}=\tau / \varepsilon$. The system (A.3) then becomes

$$
\left\{\begin{array}{l}
\frac{d x}{d \tau_{1}}=\varepsilon(\rho-x \cdot y+v \cdot z) \\
\frac{d y}{d \tau_{1}}=\sigma(1-y)-x \cdot y+k \cdot z \\
\frac{d z}{d \tau_{1}}=x \cdot y-k \cdot z
\end{array}\right.
$$

Thus, over a period of time of $\tau 1=O(1)$, we have $x\left(\tau_{1}\right) \approx 1$ and, since $y(0)=1, y$ and $z$ approximately satisfy the system

$$
\left\{\begin{array}{l}
\frac{d y}{d \tau_{1}}=-y+k \cdot z \\
\frac{d z}{d \tau_{1}}=y-k \cdot z
\end{array}\right.
$$

over this period. Therefore, in light of the initial conditions,

$y\left(\tau_{1}\right)+z\left(\tau_{1}\right)=1$ for $\tau_{1}=O(1)$

Using this equation to eliminate $y$ from the second equation of (A.6) we obtain

$\frac{d z}{d \tau_{1}}=1-(1+k) z$

and hence

$z\left(\tau_{1}\right) \rightarrow \frac{1}{1+k}$ and $y\left(\tau_{1}\right) \rightarrow \frac{k}{1+k}$ and $\quad \tau_{1} \rightarrow \infty$

In terms of the time-variable $\tau_{1}$, the half-life amounts to $\tau_{1 ; 1 / 2}=\ln (2) /(1+\kappa)$. Since $K_{\mathrm{m}}=1.1$, it follows that for the larger values of $S_{0}$ we have $\kappa=K_{\mathrm{m}} / S_{0} \ll 1$ so that $\tau_{1 ; 1 / 2} \approx \ln (2)$.
Returning to the original time variables we find that as $t \rightarrow \infty$,

$$
E S(t) \rightarrow E_{0} \frac{S_{0}}{K_{\mathrm{m}}+S_{0}}, \quad E(t) \rightarrow E_{0} \frac{K_{\mathrm{m}}}{K_{\mathrm{m}}+S_{0}} \text { and } \quad t_{1 / 2}=\frac{\ln (2)}{k_{\mathrm{on}} S_{0}}
$$

In Fig. 11, we show a blow-up of the initial behaviour of substrate and enzyme versus time. We see that, as predicted by (A.10), the half-life decreases as the initial substrate concentration $S_{0}$ increases.

\section{APPENDIX B COMPUTATION OF THE TERMINAL SLOPE}

In order to compute the terminal slope $\lambda_{\mathrm{z}}$ for the ligand concentration profile we linearise the system (1) about the steady state $(S, E, E S)=\left(0, E_{0}, 0\right)$. Writing $S=u, E=\underline{E}_{0}+v$ and $\mathrm{ES}=w$, we obtain the linear system

$\left\{\begin{array}{l}\frac{d u}{d t}=-k_{\mathrm{on}} E_{0} u+k_{\mathrm{off}} w \\ \frac{d v}{d t}=-k_{\mathrm{on}} E_{0} u-k_{\mathrm{deg}} v+k_{\mathrm{off}} w \\ \frac{d w}{d t}=k_{\mathrm{on}} E_{0} u-\left(k_{\mathrm{off}}+k_{\mathrm{cat}}\right) w\end{array}\right.$

when higher order terms are omitted. It is convenient to write this system in vector-398 and matrix notation:

$\frac{d Y}{d t}=A \cdot Y$ where $\quad Y=\left(\begin{array}{c}u \\ v \\ w\end{array}\right)$

and $A$ is the coefficient matrix of the linear system (B.1):

$A=\left(\begin{array}{ccc}-k_{\text {on }} E_{0} & 0 & k_{\text {off }} \\ -k_{\text {on }} E_{0} & -k_{\text {deg }} & k_{\text {off }} \\ k_{\text {on }} E_{0} & 0 & -\left(k_{\text {off }}+k_{\text {cat }}\right)\end{array}\right)$

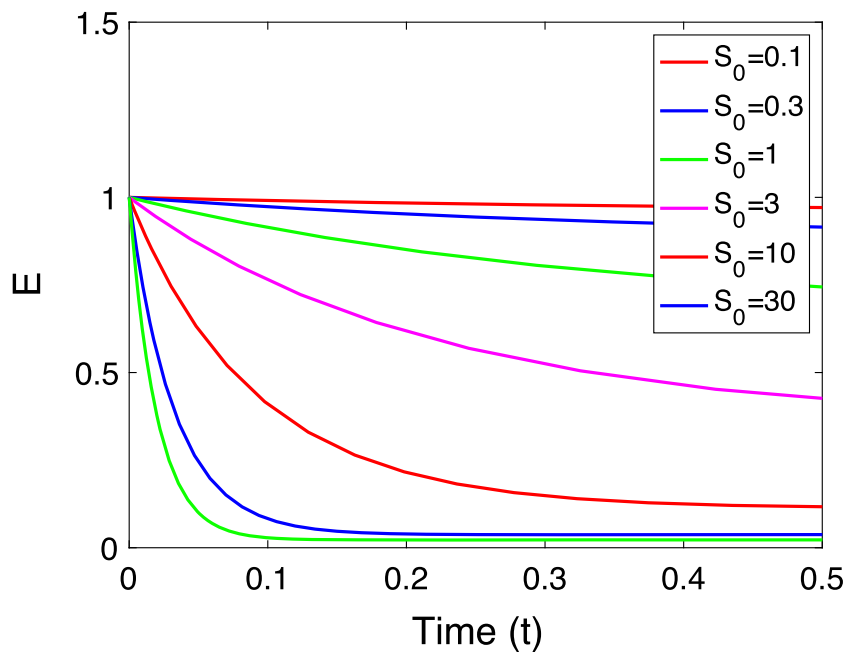

Fig. 11. Initial enzyme behaviour for seven initial substrate concentrations. The parameter values are taken from Table III 
The solution of Eq. (B.2) is then of the form

$Y(t)=C_{1} \eta_{1} e^{-\lambda 1 t}+C_{2} \eta_{2} e^{-\lambda 2 t}+C_{3} \eta_{3} e^{-\lambda 3 t}$

where $\lambda_{1}, \lambda_{2}$ and $\lambda_{3}$ are the eigenvalues of the matrix $-A$, and $\eta_{1}, \eta_{2}, \eta_{3}$ are the corresponding eigenvectors with $C_{1}, C_{2}$ and $C_{3}$ suitable constants. The eigenvalues are the roots of the equation

$\operatorname{det}(A+\lambda I)=0$

Thus, we find that

$\lambda_{1}=k_{\mathrm{deg}}$

and $\lambda_{2}$ and $\lambda_{3}$ are the roots of the quadratic equation

$\lambda^{2}-a \lambda+b=0$

where

$a=k_{\mathrm{on}} E_{0}+k_{\mathrm{off}}+k_{\mathrm{cat}}$ and $b=k_{\mathrm{on}} E_{0} k_{\mathrm{cat}}$

For the parameter values of Table III, we obtain $a=2.1$ and $b=1$, i.e. $\lambda_{1}=0.01, \lambda_{2}=1.37$ and $\lambda_{3}=0.73$. Therefore, the terminal slope $\lambda_{\mathrm{z}}$ is given by $\lambda_{\mathrm{z}}=k_{\mathrm{deg}}=0.01$.

Open Access This article is distributed under the terms of the Creative Commons Attribution 4.0 International License (http://creativecommons.org/licenses/by/4.0/), which permits unrestricted use, distribution, and reproduction in any medium, provided you give appropriate credit to the original author(s) and the source, provide a link to the Creative Commons license, and indicate if changes were made.

\section{REFERENCES}

1. Michaelis L, Menten ML. Die Kinetik der Invertinwirkung. Biochem Z. 1913;49:333-69.

2. Pelkonen O, Turpeinen M, Hakkola J, Honkakoski P, Hukkanen J, Raunio H. Inhibition and induction of human cytochrome P450 enzymes: current status. Arch Toxicol. 2008;82:667-715.

3. Mathieson T, Franken H, Kosinski J, Kurzawa N, Zinn N, Sweetman G, et al. Systematic analysis of protein turnover in primary cells. Nat Commun. 2018;9:689. https://doi.org/10.1038/ s41467-018-03106-1.

4. Cohen LD, Zuchman R, Sorokina O, Muller A, Dieterich DC, Armstrong JD, et al. Metabolic turnover of synaptic proteins: kinetics, interdependencies and implications for synaptic maintenance. PLoS One. 2013;8:1-20.

5. Fowler JS, Volkow ND, Logan J, Wang GJ, MacGregor RR, Schyler D, et al. Slow recovery of human brain MAO B after Ldeprenyl (Selegeline) withdrawal. Synapse. 1994;194(18):86-93.
6. Yang J, Liao M, Shou M, Jamei M, Rowland Yeo K, Tucker GT, et al. Cytochrome P450 turnover: regulation of synthesis and degradation, methods for determining rates, and implications for the prediction of drug interactions. Curr Drug Metab. 2008;9:384-93.

7. von Bahr C, Steiner E, Koike Y, Gabrielsson J. Time course of enzyme induction in humans: effect of pentobarbital on nortriptyline metabolism. Clin Pharmacol Ther. 1998;64:18-26.

8. Rostami-Hodjegan A. Translation of in vitro metabolic data to predict in vivo drug-drug interactions: IVIVE and modeling and simulation. In: Pang KS, Rodrigues AD, Peter RM, editors. Enzyme- and transporter-based drug-drug interactions: progress and future challenges. New York: Springer; 2010. ISBN 978-14419-0839-1.

9. Levy G. Predicting effective drug concentrations for individual patients: determinants of pharmacodynamic variability. Clin Pharmacokinet. 1998;34:323-33. https://doi.org/10.2165/ 00003088-199834040-00005.

10. Pang PS, Rodrigues AD, Peter RM, editors. Enzyme- and transporter-based drug-drug interactions: progress and future challenges. New York: Springer; 2010. ISBN 978-1-4419-0839-1

11. Pang KS, Sun H, Chow ECY. Impact of physiological determinants: flow, binding, transporters and enzymes on organ and total body clearances. In: Pang PS, Rodrigues AD, Peter RM, editors. Enzyme- and transporter-based drug-drug interactions: progress and future challenges. New York: Springer; 2010. ISBN 978-1-4419-0839-1.

12. Russel F-GM. Transporters: importance in drug absorption, distribution, and removal. In: Pang PS, Rodrigues AD, Peter RM, editors. Enzyme- and transporter based drug-drug interactions: progress and future challenges. New York: Springer; 2010. ISBN 978-1-4419-0839-1.

13. Dayneka NL, Garg V, Jusko WJ. Comparison of four basic models of indirect pharmacodynamic responses. J Pharmacokinet Biopharm. 1993;21:457-78.

14. Mager DE, Wyska E, Jusko WJ. Diversity of mechanism-based pharmacodynamic models. Drug Metab Dispos. 2003;31:510-9.

15. Gabrielsson J, Peletier LA. Pharmacokinetic steady-states highlight interesting target-mediated disposition properties. AAPS J. 2017;19(3):772-86.

16. Gabrielsson J, Peletier LA, Hjorth S. In vivo potency revisited keep the target in sight. Pharmacol Ther. 2018;835:154-161. https://doi.org/10.1016/j.pharmthera.2017.10.011.

17. Rowland M, Benet LZ, Graham GG. Clearance concepts in pharmacokinetics. J Pharmacokinet Biopharm. 1973;1:123-35.

18. Benet LZ. Clearance (née Rowland) concepts: a downdate and an update. J Pharmacokinet Pharmacodyn. 2010;37:529-39.

19. Gabrielsson J, Weiner D. Pharmacokinetic and pharmacodynamic data analysis, concept and applications. Stockholm: Sw. Pharmaceutical Press; 2016.

20. Levy RH, Dumain MS. Time-dependent kinetics VI: direct relationship be-tween equations from drug levels during induction and those involving constant clearance. J Pharm Sci. 1979;68:934-6.

21. Levy RH. In: Rowland M, Tucker G, editors. Time-dependent pharmacokinetics in: pharmacokinetics: theory and methodology. Oxford: Pergamon Press; 1986.

22. Peletier LA, Gabrielsson J. Dynamics of target-mediated drug disposition: characteristic profiles and parameter identification. J Pharmacokinet Pharmacodyn. 2012;39:429-51.

23. Geary RS, Baker BF, Crooke ST. Clinical and preclinical pharmacokinetics and pharmacodynamics of Mipomersen (Kynamro): a second-generation antisense oligonucleotide inhibitor of apolipoprotein B. Clin Pharmacokinet. 2015;54:133-46.

24. Abramson FP. Kinetic models of induction: I. Persistence of inducing agent. J Pharm Sci. 1986a;75:223-8.

25. Abramson FP. Kinetic models of induction: II. Decreased turnover of a product or its precursor. J Pharm Sci. 1986b;75:229-32.

26. Houston JB, Galetin A. In vitro techniques to study drug-drug interactions of drug metabolism: cytochrome P450. In: Pang PS, Rodrigues AD, Peter RM, editors. Enzyme- and transporterbased drug-drug interactions: Progress and Future Challenges. New York: Springer; 2010. ISBN 978-1-4419-0839-1. 